\title{
DEGREE BOUNDS FOR SEPARATING INVARIANTS
}

\author{
Martin Kohls and Hanspeter Kraft
}

\begin{abstract}
If $V$ is a representation of a linear algebraic group $G$, a set $S$ of $G$-invariant regular functions on $V$ is called separating if the following holds: If two elements $v, v^{\prime} \in V$ can be separated by an invariant function, then there is an $f \in S$ such that $f(v) \neq f\left(v^{\prime}\right)$. It is known that there always exist finite separating sets. Moreover, if the group $G$ is finite, then the invariant functions of degree $\leq|G|$ form a separating set. We show that for a non-finite linear algebraic group $G$ such an upper bound for the degrees of a separating set does not exist.

If $G$ is finite, we define $\beta_{\text {sep }}(G)$ to be the minimal number $d$ such that for every $G$ module $V$ there is a separating set of degree $\leq d$. We show that for a subgroup $H \subset G$ we have $\beta_{\text {sep }}(H) \leq \beta_{\text {sep }}(G) \leq[G: H] \cdot \beta_{\text {sep }}(H)$, and that $\beta_{\text {sep }}(G) \leq \beta_{\text {sep }}(G / H) \cdot \beta_{\text {sep }}(H)$ in case $H$ is normal. Moreover, we calculate $\beta_{\text {sep }}(G)$ for some specific finite groups.
\end{abstract}

\section{Introduction}

Let $K$ be an algebraically closed field of arbitrary characteristic. Let $G$ be a linear algebraic group and $X$ a $G$-variety, i.e. an affine variety equipped with a (regular) action of $G$, everything defined over $K$. We denote by $\mathcal{O}(X)$ the coordinate ring of $X$ and by $\mathcal{O}(X)^{G}$ the subring of $G$-invariant regular functions. The following definition is due to Derksen and Kemper [4, Definition 2.3.8].

Definition 1. Let $X$ be a $G$-variety. A subset $S \subset \mathcal{O}(X)^{G}$ of the invariant ring of $X$ is called separating (or $G$-separating) if the following holds:

For any pair $x, x^{\prime} \in X$, if $f(x) \neq f\left(x^{\prime}\right)$ for some $f \in \mathcal{O}(X)^{G}$ then there is an $h \in S$ such that $h(x) \neq h\left(x^{\prime}\right)$.

It is known and easy to see that there always exists a finite separating set (see [4, Theorem 2.3.15]).

If $V$ is a $G$-module, i.e. a finite dimensional $K$-vector space with a regular linear action of $G$, we would like to know a priory bounds for the degrees of the elements in a separating set. We denote by $\mathcal{O}(V)_{d} \subset \mathcal{O}(V)$ the homogeneous functions of degree $d$ (and the zero function), and put $\mathcal{O}(V)_{\leq d}:=\bigoplus_{i=0}^{d} \mathcal{O}(V)_{i}$.

Definition 2. For a $G$-module $V$ define

$$
\beta_{\text {sep }}(G, V):=\min \left\{d \mid \mathcal{O}(V)_{\leq d}^{G} \text { is } G \text {-separating }\right\} \in \mathbb{N},
$$

and set

$$
\beta_{\text {sep }}(G):=\sup \left\{\beta_{\text {sep }}(G, V) \mid V \text { a } G \text {-module }\right\} \in \mathbb{N} \cup\{\infty\} .
$$

The main results of this note are the following.

Received by the editors July 13, 2010. 
Theorem A. The group $G$ is finite if and only if $\beta_{\mathrm{sep}}(G)$ is finite.

In order to prove this we will show that $\beta_{\mathrm{sep}}\left(K^{+}\right)=\infty$, that $\beta_{\mathrm{sep}}\left(K^{*}\right)=\infty$, that $\beta_{\text {sep }}(G)=\infty$ for every semisimple group $G$, and that $\beta_{\text {sep }}\left(G^{0}\right) \leq \beta_{\text {sep }}(G)$ where $G^{0}$ denotes the identity component of $G$ (see Theorem 1 in section 3 ).

Theorem B. Let $G$ be a finite group and $H \subset G$ a subgroup. Then

$$
\beta_{\mathrm{sep}}(H) \leq \beta_{\mathrm{sep}}(G) \leq[G: H] \beta_{\mathrm{sep}}(H) \text {, and so } \beta_{\mathrm{sep}}(G) \leq|G| \text {. }
$$

Moreover, if $H \subset G$ is normal, then

$$
\beta_{\text {sep }}(G) \leq \beta_{\text {sep }}(G / H) \beta_{\text {sep }}(H) .
$$

This will be done in section 4 where we formulate and prove a more precise statement (Theorem 2).

Finally, we have the following explicit results for finite groups.

Theorem C. (a) Let char $K=2$. Then $\beta_{\text {sep }}\left(S_{3}\right)=4$.

(b) Let char $K=p>0$ and let $G$ be a finite p-group. Then $\beta_{\mathrm{sep}}(G)=|G|$.

(c) Let $G$ be a finite cyclic group. Then $\beta_{\mathrm{sep}}(G)=|G|$.

(d) Assume $\operatorname{char}(K)=p$ is odd, and $r \geq 1$. Then $\beta_{\text {sep }}\left(D_{2 p^{r}}\right)=2 p^{r}$.

For a reductive group $G$ one knows that the condition $f(x) \neq f\left(x^{\prime}\right)$ for some invariant $f$ (in Definition 1) is equivalent to the condition $\overline{G x} \cap \overline{G x^{\prime}}=\emptyset$, see [13, Corollary 3.5.2]. This gives rise to the following definition.

Definition 3. Let $X$ be a $G$-variety. A $G$-invariant morphism $\varphi: X \rightarrow Y$ where $Y$ is an affine variety is called separating (or $G$-separating) if the following condition holds: For any pair $x, x^{\prime} \in X$ such that $\overline{G x} \cap \overline{G x^{\prime}}=\emptyset$ we have $\varphi(x) \neq \varphi\left(x^{\prime}\right)$.

Remark 1. If $\varphi: X \rightarrow Y$ is $G$-separating and $X^{\prime} \subset X$ a closed $G$-stable subvariety, then the induced morphism $\left.\varphi\right|_{X^{\prime}}: X^{\prime} \rightarrow Y$ is also $G$-separating.

Remark 2. Choose a closed embedding $Y \subset K^{m}$ and denote by $\varphi_{1}, \ldots, \varphi_{m} \in \mathcal{O}(X)$ the coordinate functions of $\varphi: X \rightarrow Y \subset K^{m}$. If $\varphi$ is separating, then $\left\{\varphi_{1}, \ldots, \varphi_{m}\right\}$ is a separating set. The converse holds if $G$ is reductive, but not in general, as shown by the standard linear action of $K^{+}$on $K^{2}$ given by $s(x, y)=(x+s y, y)$ which does not admit a separating morphism, but has $\{y\}$ as a separating set.

\section{Some useful results}

We want to recall some facts about the $\beta_{\text {sep-values, and compare them with results }}$ for the classical $\beta$-values for generating invariants introduced by SCHMID [15]: $\beta(G)$ is the minimal $d \in \mathbb{N}$ such that, for every $G$-module $V$, the invariant ring $\mathcal{O}(V)^{G}$ is generated by the invariants of degree $\leq d$.

By Derksen and Kemper [4, Corollary 3.9.14], we have $\beta_{\text {sep }}(G) \leq|G|$. This is in perfect analogy to the Noether bound which says that $\beta(G) \leq|G|$ in the non-modular case (i.e. if $\operatorname{char}(K) \nmid|G|)$, see $[8,9,15]$. Of course we have $\beta_{\text {sep }}(G) \leq \beta(G)$, so every upper bound for $\beta(G)$ gives one for $\beta_{\text {sep }}(G)$. 
In characteristic zero and in the non-modular case there are the bounds by SCHMID [15] and by Domokos, Hegedüs, and Sezer [6, 16] which improve the Noether bound. In particular, $\beta(G) \leq \frac{3}{4}|G|$ for non-modular non-cyclic groups $G$, by [16] .

For a linear algebraic group $G$ it is shown by BRYANT, DERKSEN and KEMPER $[2,5]$ that $\beta(G)<\infty$ if and only if $G$ is finite and $p \nmid|G|$ which is the analogon to our Theorem A. For further results on degree bounds, we recommend the overview article of WEHLAU [18].

The following results will be useful in the sequel.

Proposition 1. Let $H \subset G$ be a closed subgroup, $X$ an affine $G$-variety and $Z$ an affine $H$-variety. Let $\iota: Z \rightarrow X$ be an $H$-equivariant morphism and assume that $\iota^{*}$ induces a surjection $\mathcal{O}(X)^{G} \rightarrow \mathcal{O}(Z)^{H}$. If $S \subset \mathcal{O}(X)^{G}$ is $G$-separating, then the image $\iota^{*}(S) \subset \mathcal{O}(Z)^{H}$ is $H$-separating.

Proof. Let $f \in \mathcal{O}(Z)^{H}$ and $z_{1}, z_{2} \in Z$ such that $f\left(z_{1}\right) \neq f\left(z_{2}\right)$. By assumption $f=\iota^{*}(\tilde{f})$ for some $\tilde{f} \in \mathcal{O}(X)^{G}$. Put $x_{i}:=\iota\left(z_{i}\right)$. Then $\tilde{f}\left(x_{1}\right)=f\left(z_{1}\right) \neq f\left(z_{2}\right)=\tilde{f}\left(x_{2}\right)$. Thus we can find an $h \in S$ such that $h\left(x_{1}\right) \neq h\left(x_{2}\right)$. It follows that $\bar{h}:=\iota^{*}(h) \in \iota^{*}(S)$ and $\bar{h}\left(z_{1}\right)=h\left(x_{1}\right) \neq h\left(x_{2}\right)=\bar{h}\left(z_{2}\right)$.

Remark 3. In general, the inverse map $\left(\iota^{*}\right)^{-1}$ does not take $H$-separating sets to $G$-separating sets. Take $K^{+} \subset \mathrm{SL}_{2}$ as the subgroup of upper triangular unipotent matrices, $X=K^{2} \oplus K^{2} \oplus K^{2}$ the sum of three copies of the standard representation of $\mathrm{SL}_{2}$ and $Z=K^{2} \oplus K^{2}$ the sum of two copies of the standard representation of $K^{+}$. Then $\iota: Z \rightarrow X,(v, w) \mapsto((1,0), v, w)$ is $K^{+}$-equivariant and induces an isomorphism $\mathcal{O}(X)^{\mathrm{SL}_{2}} \stackrel{\sim}{\longrightarrow} \mathcal{O}(Z)^{K^{+}}$(see [14]). In fact, choosing the coordinates $\left(x_{0}, x_{1}, y_{0}, y_{1}, z_{0}, z_{1}\right)$ on $X$ and $\left(y_{0}, y_{1}, z_{0}, z_{1}\right)$ on $Y$, we get from the classical description [3] of the invariants and covariants of copies of $K^{2}$ :

$$
\begin{aligned}
\mathcal{O}(X)^{\mathrm{SL}_{2}(K)} & =K\left[y_{1} x_{0}-y_{0} x_{1}, z_{1} x_{0}-z_{0} x_{1}, y_{1} z_{0}-y_{0} z_{1}\right], \\
\mathcal{O}(Y)^{K^{+}} & =K\left[y_{1}, z_{1}, y_{1} z_{0}-y_{0} z_{1}\right],
\end{aligned}
$$

and the claim follows, because $\iota^{*}\left(x_{0}\right)=1, \iota^{*}\left(x_{1}\right)=0$.

Now take $S:=\left\{y_{1}, z_{1}, y_{1}\left(y_{1} z_{0}-y_{0} z_{1}\right), z_{1}\left(y_{1} z_{0}-y_{0} z_{1}\right)\right\} \subset \mathcal{O}(Z)^{K^{+}}$. We claim that $S$ is a $K^{+}$-separating set, but $\left(\iota^{*}\right)^{-1}(S) \subset \mathcal{O}(X)^{\mathrm{SL}_{2}}$ is not $\mathrm{SL}_{2}$-separating. For the first claim one has to use that if $y_{1}$ and $z_{1}$ both vanish, then the third generator $y_{1} z_{0}-y_{0} z_{1}$ of the invariant $\operatorname{ring} \mathcal{O}(Y)^{K^{+}}$also vanishes. For the second claim we consider the elements $v=((0,0),(0,0),(0,0))$ and $v^{\prime}=((0,0),(1,0),(0,1))$ of $X$, which are separated by the invariants, but not by $\left(\iota^{*}\right)^{-1}(S)$.

For the following application recall that for a closed subgroup $H \subset G$ of finite index the induced module $\operatorname{Ind}_{H}^{G} V$ of an $H$-module $V$ is a finite dimensional $G$-module.

Corollary 1. Let $H \subset G$ be a closed subgroup of finite index and let $V$ be an $H$ module. Then $\beta_{\text {sep }}(H, V) \leq \beta_{\text {sep }}\left(G, \operatorname{Ind}_{H}^{G} V\right)$. In particular, $\beta_{\text {sep }}(H) \leq \beta_{\text {sep }}(G)$.

Proof. By definition, $\operatorname{Ind}_{H}^{G} V$ contains $V$ as an $H$-submodule in a canonical way. If $n:=[G: H]$ and $G=\bigcup_{i=1}^{n} g_{i} H$, then $\operatorname{Ind}_{H}^{G} V=\bigoplus_{i=1}^{n} g_{i} V$. Moreover, the inclusion $\iota: V \hookrightarrow \operatorname{Ind}_{H}^{G} V$ induces a surjection $\iota^{*}: \mathcal{O}\left(\operatorname{Ind}_{H}^{G}(V)\right)^{G} \rightarrow \mathcal{O}(V)^{H},\left.f \mapsto f\right|_{V}$. In fact, for $f \in \mathcal{O}(V)_{+}^{H}$, a preimage $\tilde{f}$ is given by $\tilde{f}\left(g_{1} v_{1}, \ldots, g_{n} v_{n}\right):=\sum_{i=1}^{n} f\left(v_{i}\right), v_{i} \in V$, 
which is easily seen to be $G$-invariant. Now the claim follows from Proposition 1 above, because the restriction map $\iota^{*}$ is linear and so preserves degrees.

Proposition 2 (DERKSEN and Kemper [4, Theorem 2.3.16]). Let $G$ be a reductive group, $V$ a $G$-module und $U \subset V$ a submodule. The restriction map $\mathcal{O}(V) \rightarrow \mathcal{O}(U)$, $\left.f \mapsto f\right|_{U}$ takes every separating set of $\mathcal{O}(V)^{G}$ to a separating set of $\mathcal{O}(U)^{G}$. In particular, we have

$$
\beta_{\mathrm{sep}}(G, U) \leq \beta_{\mathrm{sep}}(G, V) .
$$

Let us mention here that in positive characteristic the restriction map is in general not surjective when restriced to the invariants, and so a generating set is not necessarily mapped onto a generating set.

We finally remark that for finite groups there always exist $G$-modules $V$ such that $\beta_{\text {sep }}(G, V)=\beta_{\text {sep }}(G)$. The same holds for the $\beta$-values in characteristic zero.

Proposition 3. Let $G$ be a finite group group and $V_{\text {reg }}=K G$ its regular representation. Then

$$
\beta_{\text {sep }}(G)=\beta_{\text {sep }}\left(G, V_{\text {reg }}\right) .
$$

In fact, every $G$-module $V$ can be embedded as a submodule into $V_{\text {reg }}^{\operatorname{dim}} V$. Since, by [7, Corollary 3.7], $\beta_{\text {sep }}\left(G, V^{m}\right)=\beta_{\text {sep }}(G, V)$ for any $G$-module $V$ and every positive integer $m$, the claim follows from Proposition 2.

\section{The case of non-finite algebraic groups}

In this section we prove the following theorem which is equivalent to Theorem $\mathrm{A}$ from the first section.

Theorem 1. For any non-finite linear algebraic group $G$ we have $\beta_{\text {sep }}(G)=\infty$.

We start with the additive group $K^{+}$. Denote by $V=K e_{0} \oplus K e_{1} \simeq K^{2}$ the standard 2-dimensional $K^{+}$-module: $s \cdot e_{0}:=e_{0}, s \cdot e_{1}:=s e_{0}+e_{1}$ for $s \in K^{+}$. If char $K=p>0$ we can "twist" the module $V$ with the Frobenius map $F^{n}: K^{+} \rightarrow K^{+}, s \mapsto s^{p^{n}}$ to obtain another $K^{+}$-module which we denote by $V_{F^{n}}$.

Proposition 4. Let char $K=p>0$ and consider the $K^{+}$-module $W:=V \oplus V_{F^{n}}$. We write $\mathcal{O}(W)=K\left[x_{0}, x_{1}, y_{0}, y_{1}\right]$. Then $\mathcal{O}(W)^{K^{+}}=K\left[x_{1}, y_{1}, x_{0}^{p^{n}} y_{1}-x_{1}^{p^{n}} y_{0}\right]$. In particular, $\beta_{\mathrm{sep}}\left(K^{+}, W\right)=p^{n}+1$ and so $\beta_{\mathrm{sep}}\left(K^{+}\right)=\infty$.

Proof. It is easy to see that $f:=x_{0}^{p^{n}} y_{1}-x_{1}^{p^{n}} y_{0}$ is $K^{+}$-invariant. Define the $K^{+}$invariant morphism

$$
\pi: W \rightarrow K^{3}, \quad w=\left(a_{0}, a_{1}, b_{0}, b_{1}\right) \mapsto\left(a_{1}, b_{1}, a_{0}^{p^{n}} b_{1}-a_{1}^{p^{n}} b_{0}\right) .
$$

Over the affine open set $U:=\left\{\left(c_{1}, c_{2}, c_{3}\right) \in K^{3} \mid c_{1} \neq 0\right\}$, the induced map $\pi^{-1}(U) \rightarrow$ $U$ is a trivial $K^{+}$-bundle. In fact, the morphism $\rho: U \rightarrow \pi^{-1}(U)$ given by $\left(c_{1}, c_{2}, c_{3}\right) \mapsto$ $\left(0, c_{1},-c_{1}^{-p^{n}} c_{3}, c_{2}\right)$ is a section of $\pi$, inducing a $K^{+}$-equivariant isomorphism $K^{+} \times$ $U \stackrel{\sim}{\longrightarrow} \pi^{-1}(U),(s, u) \mapsto s \cdot \rho(u)$. This implies that $\mathcal{O}(W)_{x_{1}}^{K^{+}}=K\left[x_{1}, x_{1}^{-1}, y_{1}, f\right]$, hence $\mathcal{O}(W)^{K^{+}}=K\left[x_{0}, x_{1}, y_{0}, y_{1}\right] \cap K\left[x_{1}, x_{1}^{-1}, y_{1}, f\right]$, and the claim follows easily. 
If $K$ has characteristic zero, we need a different argument. Denote by $V_{n}:=S^{n} V$ the $n$th symmetric power of the standard $K^{+}$-module $V=K e_{0} \oplus K e_{1}$ (see above). This module is cyclic of dimension $n+1$, i.e. $V_{n}=\left\langle K^{+} v_{n}\right\rangle$ where $v_{n}:=e_{1}^{n}$, and for any $s \in K^{+}, s \neq 0$, the endomorphism $v \mapsto s v-v$ of $V_{n}$ is nilpotent of rank $n$. In particular, $V_{n}^{K^{+}}=K v_{0}$ where $v_{0}:=e_{0}^{n} \in V_{n}$.

Remark 4 . For $q \geq 1$ consider the $q$ th symmetric power $S^{q} V_{n}$ of the module $V_{n}$. Then the cyclic submodule $\left\langle K^{+} v_{n}^{q}\right\rangle \subset S^{q} V_{n}$ generated by $v_{n}^{q}$ is $K^{+}$-isomorphic to $V_{q n}$, and $\left\langle K^{+} v_{n}^{q}\right\rangle^{K^{+}}=K v_{0}^{q}$. One way to see this is by remarking that the modules $V_{n}$ are $\mathrm{SL}_{2}(K)$-modules in a natural way, and then to use representation theory of $\mathrm{SL}_{2}(K)$.

Proposition 5. Let char $K=0$. Consider the $K^{+}{ }_{\text {-module }} W=V^{*} \oplus V_{n}$ and the two vectors $w:=\left(x_{0}, v_{0}\right)$ and $w^{\prime}:=\left(x_{0}, 0\right)$ of $W$. Then there is a $K^{+}$-invariant function $f \in \mathcal{O}(W)^{K^{+}}$separating $w$ and $w^{\prime}$, and any such $f$ has degree $\operatorname{deg} f \geq n+1$. In particular, $\beta_{\mathrm{sep}}\left(K^{+}, W\right) \geq n+1$, and so $\beta_{\mathrm{sep}}\left(K^{+}\right)=\infty$.

Proof. Let $U_{1}, U_{2}$ be two finite dimensional vector spaces. There is a canonical isomorphism

$$
\Psi: \mathcal{O}\left(U_{1}^{*} \oplus U_{2}\right)_{(p, q)} \stackrel{\sim}{\longrightarrow} \operatorname{Hom}\left(S^{q} U_{2}, S^{p} U_{1}\right)
$$

where $\mathcal{O}\left(U_{1}^{*} \oplus U_{2}\right)_{(p, q)}$ denotes the subspace of those regular functions on $U_{1}^{*} \oplus U_{2}$ which are bihomogeneous of degree $(p, q)$. If $F=\Psi(f)$, then for any $x \in U_{1}^{*}$ and $u \in U_{2}$ we have

$$
f(x, u)=x^{p}\left(F\left(u^{q}\right)\right) .
$$

(Since we are in characteristic 0 we can identify $S^{p}\left(U_{1}^{*}\right)$ with $\left(S^{p} U_{1}\right)^{*}$.) Moreover, if $U_{1}, U_{2}$ are $G$-modules, then $\Psi$ is $G$-equivariant and induces an isomorphism between the $G$-invariant bihomogeneous functions and the $G$-linear homomorphisms:

$$
\Psi: \mathcal{O}\left(U_{1}^{*} \oplus U_{2}\right)_{(p, q)}^{G} \stackrel{\sim}{\longrightarrow} \operatorname{Hom}_{G}\left(S^{q} U_{2}, S^{p} U_{1}\right) .
$$

For the $K^{+}$-module $W=V^{*} \oplus V_{n}$ we thus obtain an isomorphism

$$
\Psi: \mathcal{O}\left(V^{*} \oplus V_{n}\right)_{(p, q)}^{K^{+}} \stackrel{\sim}{\longrightarrow} \operatorname{Hom}_{K^{+}}\left(S^{q} V_{n}, S^{p} V\right) .
$$

Putting $p=n$ and $q=1$ and defining $f \in \mathcal{O}\left(V^{*} \oplus V_{n}\right)_{(n, 1)}^{K^{+}}$by $\Psi(f)=\operatorname{Id}_{V_{n}}$, we get $f(w)=f\left(x_{0}, v_{0}\right)=x_{0}^{n}\left(v_{0}\right)=x_{0}^{n}\left(e_{0}^{n}\right) \neq 0$, and $f\left(w^{\prime}\right)=f\left(x_{0}, 0\right)=0$. Hence $w$ and $w^{\prime}$ can be separated by invariants.

Now let $f$ be a $K^{+}$-invariant separating $w$ and $w^{\prime}$ where $\operatorname{deg} f=d$. We can clearly assume that $f$ is bihomogeneous, say of degree $(p, q)$ where $p+q=d$. Because $f$ must depend on $V_{n}$, we have $q \geq 1$. Hence $f\left(w^{\prime}\right)=f\left(x_{0}, 0\right)=0$, and so $f(w)=f\left(x_{0}, v_{0}\right) \neq$ 0 . This implies for $F:=\Psi(f)$ that $F\left(v_{0}^{q}\right) \neq 0$. Now it follows from Remark 4 above that $F$ induces an injective map of $\left\langle K^{+} v_{n}^{q}\right\rangle$ into $S^{p} V$, and so

$$
p+1=\operatorname{dim} S^{p} V \geq \operatorname{dim}\left\langle K^{+} v_{n}^{q}\right\rangle=q n+1 \geq n+1 .
$$

Hence $\operatorname{deg} f=p+q \geq n+1$.

To handle the general case we use the following construction. Let $G$ be an algebraic group and $H \subset G$ a closed subgroup. We assume that $H$ is reductive. For an affine $H$-variety $X$ we define

$$
G \times{ }^{H} X:=(G \times X) / / H:=\operatorname{Spec}\left(\mathcal{O}(G \times X)^{H}\right)
$$


where $H$ acts (freely) on the product $G \times X$ by $h(g, x):=\left(g h^{-1}, h x\right)$, commuting with the action of $G$ by left multiplication on the first factor. We denote by $[g, x]$ the image of $(g, x) \in G \times X$ in the quotient $G \times{ }^{H} X$.

The following is well-known. It follows from general results from geometric invariant theory, see e.g. [12].

(a) The canonical morphism $G \times{ }^{H} X \rightarrow G / H,[g, x] \mapsto g H$, is a fiber bundle (in the étale topology) with fiber $X$.

(b) If the action of $H$ on $X$ extends to an action of $G$, then $G \times{ }^{H} X \stackrel{\sim}{\longrightarrow} G / H \times X$ where $G$ acts diagonally on $G / H \times X$ (i.e. the fiber bundle is trivial).

(c) The canonical morphism $\iota: X \hookrightarrow G \times{ }^{H} X$ given by $x \mapsto[e, x]$ is an $H$ equivariant closed embedding.

Lemma 1. If $\varphi: G \times{ }^{H} X \rightarrow Y$ is $G$-separating, then the composite morphism $\varphi \circ$ $\iota: X \rightarrow Y$ is $H$-separating. Moreover, if $S \subset \mathcal{O}\left(G \times{ }^{H} X\right)^{G}$ is a $G$-separating set, then its image $\iota^{*}(S) \subset \mathcal{O}(X)^{H}$ is H-separating.

Proof. For $x \in X$ we have $\overline{G[e, x]}=[G, \overline{H x}]$. Therefore, if $\overline{H x} \cap \overline{H x^{\prime}}=\emptyset$, then $\overline{G[e, x]} \cap \overline{G\left[e, x^{\prime}\right]}=\emptyset$ and so $\varphi \circ \iota(x)=\varphi([e, x]) \neq \varphi\left(\left[e, x^{\prime}\right]\right)=\varphi \circ \iota\left(x^{\prime}\right)$. The second claim follows from Proposition 1, because $\mathcal{O}\left(G \times{ }^{H} X\right)^{G}=\mathcal{O}(G \times X)^{G \times H}=\mathcal{O}(X)^{H}$ and so $\iota^{*}$ induces an isomorphism $\mathcal{O}\left(G \times{ }^{H} X\right)^{G} \stackrel{\sim}{\longrightarrow} \mathcal{O}(X)^{H}$.

Now let $V$ be a $G$-module and $X:=\left.V\right|_{H}$, the underlying $H$-module. Let $H$ act on $G$ by right-multiplication with the inverse. As $H$ is reductive, the categorical quotient $G / / H$ exists as an affine $G$-variety, and can be identified with the set of left cosets $G / H$ (see [17, Exercise 5.5.9 (8)]). Choose a closed $G$-equivariant embedding $G / H \stackrel{\sim}{\longrightarrow} G w_{0} \hookrightarrow W$ where $W$ is a $G$-module (see [4, Lemma A.1.9]). Then we get the following composition of closed embeddings where the first one is $H$-equivariant and the remaining are $G$-equivariant:

$$
\mu:\left.V\right|_{H} \hookrightarrow G \times{ }^{H} V \stackrel{\sim}{\longrightarrow} G / H \times V \hookrightarrow W \times V .
$$

The map $\mu$ is given by $\mu(v)=\left(w_{0}, v\right)$. It follows from Lemma 1 and Remark 1 that for any $G$-separating morphism $\varphi: W \times V \rightarrow Y$ the composition $\varphi \circ \mu:\left.V\right|_{H} \rightarrow Y$ is $H$-separating. In particular, if $G$ is reductive, then for any $G$-separating set $S \subset$ $\mathcal{O}(W \times V)$ the image $\mu^{*}(S) \subset \mathcal{O}(V)^{H}$ is $H$-separating. Since $\operatorname{deg} \mu^{*}(f) \leq \operatorname{deg} f$ this implies the following result.

Proposition 6. Let $G$ be a reductive group, $H \subset G$ a closed reductive subgroup and $V^{\prime}$ an $H$-module. If $V^{\prime}$ is isomorphic to an $H$-submodule of a $G$-module $V$, then

$$
\beta_{\text {sep }}\left(H, V^{\prime}\right) \leq \beta_{\text {sep }}(G) .
$$

Now we can prove the main result of this section,

Proof of Theorem 1. By Corollary 1 we can assume that $G$ is connected.

(a) Let $G$ be semisimple, $T \subset G$ a maximal torus and $B \supset T$ a Borel subgroup. If $\lambda \in X(T)$ is dominant we denote by $E^{\lambda}$ the Weyl-module of $G$ of highest weight $\lambda$, and by $D^{\lambda} \subset E^{\lambda}$ the highest weight line. Choose a one-parameter subgroup $\rho: K^{*} \rightarrow T$ and define $k_{0} \in \mathbb{Z}$ by $\rho(t) u=t^{k_{0}} \cdot u$ for $u \in D^{\lambda}$. For any $n \in \mathbb{N}$ put

$$
V_{n}^{\prime}:=\left(D^{\lambda}\right)^{*} \oplus D^{n \lambda} \subset V_{n}:=\left(E^{\lambda}\right)^{*} \oplus E^{n \lambda} .
$$


Then $V_{n}^{\prime}$ is a two-dimensional $K^{*}$-module with weights $\left(-k_{0}, n k_{0}\right)$. Hence $\mathcal{O}\left(V_{n}^{\prime}\right)^{K^{*}}$ is generated by a homogeneous invariant of degree $n+1$ and so $\beta_{\text {sep }}\left(K^{*}, V_{n}^{\prime}\right)=n+1$. Now Proposition 6 implies

$$
n+1=\beta_{\mathrm{sep}}\left(K^{*}, V_{n}^{\prime}\right) \leq \beta_{\mathrm{sep}}(G)
$$

and the claim follows. In addition, we have also shown that $\beta_{\mathrm{sep}}\left(K^{*}\right)=\infty$.

(b) If $G$ admits a non-trivial character $\chi: G \rightarrow K^{*}$ then the claim follows because $\beta_{\text {sep }}(G) \geq \beta_{\text {sep }}\left(K^{*}\right)=\infty$, as we have seen in (a).

(c) If the character group of $G$ is trivial, then either $G$ is unipotent or there is a surjective homomorphism $G \rightarrow H$ where $H$ is semisimple (use [17, Corollary 8.1.6 (ii)]). In the first case there is a surjective homomorphism $G \rightarrow K^{+}$and the claim follows from Proposition 4 and Proposition 5. In the second case the claim follows from (a).

\section{Relative degree bounds}

In this section all groups are finite. We want to prove the following result which covers Theorem B from the first section.

Theorem 2. Let $G$ be a finite group, $H \subset G$ a subgroup, $V$ a $G$-module and $W$ an $H$-module. Then

$$
\beta_{\text {sep }}(H, W) \leq \beta_{\text {sep }}\left(G, \operatorname{Ind}_{H}^{G} W\right) \text { and } \beta_{\text {sep }}(G, V) \leq[G: H] \beta_{\text {sep }}(H, V) .
$$

In particular

$$
\beta_{\text {sep }}(H) \leq \beta_{\text {sep }}(G) \leq[G: H] \beta_{\text {sep }}(H) \text {, and so } \beta_{\text {sep }}(G) \leq|G| \text {. }
$$

Moreover, if $H \subset G$ is normal, then

$$
\beta_{\text {sep }}(G) \leq \beta_{\text {sep }}(G / H) \beta_{\text {sep }}(H) .
$$

Note that the inequalities $\beta_{\text {sep }}(G, V) \leq[G: H] \beta_{\text {sep }}(H, V)$ and $\beta_{\text {sep }}(G) \leq|G|$ were already proved by Derksen and Kemper ([11, Corollary 24], [4, Corollary 3.9.14]).

The proof needs some preparation. Let $V, W$ be finite dimensional vector spaces and $\varphi: V \rightarrow W$ a morphism, i.e. a polynomial map.

Definition 4. The degree of $\varphi$ is defined in the following way, generalizing the degree of a polynomial function. Choose a basis $\left(w_{1}, \ldots, w_{m}\right)$ of $W$, so that $\varphi(v)=$ $\sum_{j=1}^{m} f_{j}(v) w_{j}$ for $v \in V$. Then

$$
\operatorname{deg} \varphi:=\max \left\{\operatorname{deg} f_{j} \mid \quad j=1, \ldots, m\right\} .
$$

It is easy to see that this is independent of the choice of a basis.

If $V$ is a $G$-module and $\varphi: V \rightarrow W$ a separating morphism, then $\beta_{\text {sep }}(G, V) \leq$ $\operatorname{deg} \varphi$. Moreover, there is a separating morphism $\varphi: V \rightarrow W$ for some $W$ such that $\beta_{\text {sep }}(G, V)=\operatorname{deg} \varphi$.

For any (finite dimensional) vector space $W$ we regard $W^{d}=W \otimes K^{d}$ as the direct sum of $\operatorname{dim} W$ copies of the standard $\mathcal{S}_{d}$-module $K^{d}$. In this case we have the following result due to Draisma, Kemper and Wehlau [7, Theorem 3.4]. 
Lemma 2. The polarizations of the elementary symmetric functions form an $\mathcal{S}_{d^{-}}$

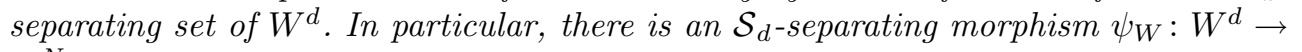
$K^{N}$ of degree $\leq d$.

Recall that the polarizations of a function $f \in \mathcal{O}(U)$ to $n$ copies of $U$ are defined in the following way. Write

$$
f\left(t_{1} u_{1}+t_{2} u_{2}+\cdots+t_{n} u_{n}\right)=\sum_{i_{1}, i_{2}, \ldots, i_{n}} t_{1}^{i_{1}} t_{2}^{i_{2}} \cdots t_{n}^{i_{n}} f_{i_{1} i_{2} \ldots i_{n}}\left(u_{1}, u_{2}, \ldots, u_{n}\right)
$$

Then the functions $f_{i_{1} i_{2} \ldots i_{n}}\left(u_{1}, u_{2}, \ldots, u_{n}\right) \in \mathcal{O}\left(U^{n}\right)$ are called polarizations of $f$. Clearly, $\operatorname{deg} f_{i_{1} i_{2} \ldots i_{n}} \leq \operatorname{deg} f$. Moreover, if $U$ is a $G$-module and $f$ a $G$-invariant, then all $f_{i_{1} i_{2} \ldots i_{n}}$ are $G$-invariants with respect to the diagonal action of $G$ on $U^{n}$.

Proof of Theorem 2. The first inequality $\beta_{\text {sep }}(H, W) \leq \beta_{\text {sep }}\left(G, \operatorname{Ind}_{H}^{G} W\right)$ is shown in Corollary 1.

Let $V$ be a $G$-module, $v, w \in V$, and let $\varphi: V \rightarrow W$ be an $H$-separating morphism of degree $\beta_{\text {sep }}(H, V)$. Consider the partition of $G$ into $H$-right cosets: $G=\bigcup_{i=1}^{d} H g_{i}$ where $d:=[G: H]$. Define the following morphism

$$
\bar{\varphi}: V \stackrel{\tilde{\varphi}}{\longrightarrow} W^{d} \stackrel{\psi_{W}}{\longrightarrow} K^{N}
$$

where $\tilde{\varphi}(v):=\left(\varphi\left(g_{1} v\right), \ldots, \varphi\left(g_{d} v\right)\right)$ and $\psi_{W}: W^{d} \rightarrow K^{N}$ is the separating morphism from Lemma 2.

We claim that $\bar{\varphi}$ is $G$-separating. In fact, for $g \in G$ define the permutation $\sigma \in$ $\mathcal{S}_{d}$ by $H g_{i} g=H g_{\sigma(i)}$, i.e. $g_{i} g=h_{i} g_{\sigma(i)}$ for a suitable $h_{i} \in H$. Then $\varphi\left(g_{i} g v\right)=$ $\varphi\left(h_{i} g_{\sigma(i)} v\right)=\varphi\left(g_{\sigma(i)} v\right)$ and so $\tilde{\varphi}(g v)=\sigma^{-1} \tilde{\varphi}(v)$. This shows that $\bar{\varphi}$ is $G$-invariant.

Assume now that $g v \neq w$ for all $g \in G$. This implies that $h g_{i} v \neq w$ for all $h \in H$ and $i=1, \ldots d$, and so $\varphi\left(g_{i} v\right) \neq \varphi(w)$ for $i=1, \ldots, d$, because $\varphi$ is $H$-separating. As a consequence, $\tilde{\varphi}(v) \neq \sigma \tilde{\varphi}(w)$ for all permutations $\sigma \in \mathcal{S}_{d}$, hence $\bar{\varphi}(v) \neq \bar{\varphi}(w)$, because $\psi_{W}$ is $\mathcal{S}_{d^{-}}$-separating, and so $\bar{\varphi}$ is $G$-separating.

For the degree we get $\operatorname{deg} \bar{\varphi} \leq \operatorname{deg} \psi_{W} \cdot \operatorname{deg} \tilde{\varphi} \leq d \cdot \operatorname{deg} \varphi=[G: H] \beta_{\operatorname{sep}}(H, V)$. This shows that

$$
\beta_{\text {sep }}(G, V) \leq[G: H] \beta_{\text {sep }}(H, V) .
$$

If $H \subset G$ is normal we can find an $H$-separating morphism $\varphi: V \rightarrow W$ of degree $\beta_{\text {sep }}(H, V)$ such that $W$ is a $G / H$-module and $\varphi$ is $G$-equivariant. Now choose an $G / H$-separating morphism $\psi: W \rightarrow U$ of degree $\beta_{\text {sep }}(G / H, W)$. Then the composition $\psi \circ \varphi: V \rightarrow U$ is $G$-separating of degree $\leq \operatorname{deg} \psi \cdot \operatorname{deg} \varphi$. Thus

$$
\beta_{\text {sep }}(G, V) \leq \beta_{\text {sep }}(G / H, W) \beta_{\text {sep }}(H, V) \leq \beta_{\text {sep }}(G / H) \beta_{\text {sep }}(H),
$$

and the claim follows.

\section{Degree bounds for some finite groups}

In principle, Proposition 3 allows to compute $\beta_{\text {sep }}(G)$ for any finite group $G$. Unfortunately, the invariant ring $\mathcal{O}\left(V_{\text {reg }}\right)^{G}$ does not behave well in a computational sense. We have been able to compute $\beta_{\text {sep }}(G)$ with Magma [1] and the algorithm of [10] in just one case (computation time about 20 minutes): 
Proposition 7 (Magma and Proposition 3). Let char $K=2$. Then $\beta_{\text {sep }}\left(S_{3}\right)=4$.

Proposition 8. Let char $K=p>0$ and let $G$ be a p-group. Then $\beta_{\mathrm{sep}}(G)=|G|$.

Proof. Let us start with a general remark. Let $G$ be an arbitrary finite group, and let $V$ be a permutation module of $G$, i.e. there is a basis $\left(v_{1}, v_{2}, \ldots, v_{n}\right)$ of $V$ which is permuted under $G$. Then the invariants are linearly spanned by the orbit sums $s_{m}$ of the monomials $m=x_{1}^{i_{1}} x_{2}^{i_{2}} \cdots x_{n}^{i_{n}} \in \mathcal{O}(V)=K\left[x_{1}, x_{2}, \ldots, x_{n}\right]$ which are defined in the usual way:

$$
s_{m}:=\sum_{f \in G m} f
$$

The value of $s_{m}$ on the fixed point $v:=v_{1}+v_{2}+\cdots+v_{n} \in V$ equals $|G m|$. Hence, $s_{m}(v)=0$ if $p$ divides the index $\left[G: G_{m}\right]$ of the stabilizer $G_{m}$ of $m$ in $G$. It follows that for a $p$-group $G$ we have $s_{m}(v) \neq 0$ if and only if $m$ is invariant under $G$.

If, in addition, $G$ acts transitively on the basis $\left(v_{1}, v_{2}, \ldots, v_{n}\right)$, then an invariant monomial $m$ is a power of $x_{1} x_{2} \cdots x_{n}$, and thus has degree $\ell n \geq \operatorname{dim} V$. If we apply this to the regular representation, the claim follows.

With Corollary 1 we get the next result.

Corollary 2. Let char $K=p>0$ and $G$ be a group of order $r p^{k}$ with $(r, p)=1$. Then $\beta_{\text {sep }}(G) \geq p^{k}$.

Proposition 9. Let $G$ be a cyclic group. Then $\beta_{\mathrm{sep}}(G)=|G|$.

Proof. Let $|G|=r p^{k}$ where $(r, p)=1, p=\operatorname{char} K$, and choose two elements $g, h \in G$ of order $r$ and $q:=p^{k}$, respectively, so that $G=\langle g, h\rangle$. We define a linear action of $G$ on $V:=\bigoplus_{i=1}^{q} K v_{i}$ by

$$
g v_{i}:=\zeta \cdot v_{i} \text { and } h v_{i}:=v_{i+1} \text { for } i=1, \ldots, q
$$

where $\zeta \in K$ is a primitive $r$ th root of unity and $v_{q+1}:=v_{1}$. We claim that the $G$ invariants $\mathcal{O}(V)^{G}$ are linearly spanned by the orbit sums $s_{m}$ where $r \mid \operatorname{deg} m$. In fact, $\mathcal{O}(V)^{\langle g\rangle}$ is linearly spanned by the monomials of degree $\ell r(\ell \geq 0)$, and the subgroup $H:=\langle h\rangle \subset G$ permutes these monomials.

Now look again at the element $v:=v_{1}+v_{2}+\cdots+v_{q} \in V$. If $r \mid \operatorname{deg} m$ then $s_{m}(v)=|H m|$, and this is non-zero if and only if the monomial $m$ is invariant under $H$. This implies that $m$ is a power of $x_{1} x_{2} \cdots x_{q}$. Since the degree of $m$ is also a multiple of $r$ we finally get $\operatorname{deg} s_{m} \geq r q=|G|$.

Corollary 3. Let $G$ be a finite group. Then we have

$$
\beta_{\text {sep }}(G) \geq \max _{g \in G}(\text { ord } g) .
$$

Let $D_{2 n}=\langle\sigma, \rho\rangle$ denote the dihedral group of order $2 n$ with $\operatorname{ord}(\sigma)=2, \operatorname{ord}(\rho)=n$ and $\sigma \rho \sigma^{-1}=\rho^{-1}$.

Proposition 10. Assume that $\operatorname{char}(K)=p$ is an odd prime, and let $r \geq 1$. Then $\beta_{\text {sep }}\left(D_{2 p^{r}}\right)=2 p^{r}$.

Note that if $\operatorname{char}(K)=p=2$, then $D_{2 p^{r}}$ is a 2 -group, so $\beta_{\mathrm{sep}}\left(D_{2^{r+1}}\right)=2^{r+1}$ by Proposition 8. We conjecture that for $\operatorname{char}(K)=2$ and $p$ an odd prime, we have $\beta_{\text {sep }}\left(D_{2 p}\right)=p+1$, which would fit with Proposition 7 . 
Proof. Put $q=p^{r}$ and define a linear action of $D_{2 p^{r}}$ on $V:=\bigoplus_{i=0}^{q-1} K v_{i}$ by

$$
\rho v_{i}=v_{i+1} \text { and } \sigma v_{i}=-v_{-i} \text { for } i=0,1, \ldots, q-1
$$

where $v_{j}=v_{i}$ if $j \equiv i \bmod q$ for $i, j \in \mathbb{Z}$. As before, the invariants under $H:=\langle\rho\rangle$ are linearly spanned by the orbit sums $s_{m}:=\sum_{f \in H m} f$ of the monomials $m=$ $x_{0}^{i_{0}} x_{1}^{i_{1}} \cdots x_{q-1}^{i_{q-1}} \in \mathcal{O}(V)=K\left[x_{0}, x_{1}, \ldots, x_{q-1}\right]$. Thus, the $D_{2 p^{r} \text {-invariants are linearly }}$ spanned by the functions $\left\{s_{m}+\sigma s_{m} \mid m\right.$ a monomial $\}$.

For $v:=v_{0}+v_{1}+\cdots+v_{q-1}$ we get $\sigma s_{m}(v)=s_{m}(\sigma v)=(-1)^{\operatorname{deg} m} s_{m}(v)$. Therefore, $s_{m}+\sigma s_{m}$ is non-zero on $v$ if and only if $s_{m}(v) \neq 0$ and the degree of $m$ is even. As in the proof of Proposition 9, $s_{m}(v) \neq 0$ implies that $m$ is a power of $x_{0} x_{1} \cdots x_{q-1}$ which has to be an even power since $q$ is odd. Thus, for $m:=\left(x_{0} x_{1} \cdots x_{q-1}\right)^{2}, s_{m}+\sigma s_{m}=2 m$ is an invariant of smallest possible degree, namely $2 q$, which does not vanish on $v$.

Let $I_{H}:=\mathcal{O}(V)_{+}^{G} \mathcal{O}(V)$ denote the Hilbert-ideal, i.e. the ideal in $\mathcal{O}(V)$ generated by all homogeneous invariants of positive degree. It is conjectured by DERKSEN and KEMPER that $I_{H}$ is generated by invariants of positive degree $\leq|G|$, see [4, Conjecture 3.8.6 (b)]. The following corollary shows that this conjectured bound can not be sharpened in general.

Corollary 4. Let char $K=p$ and $G$ a p-group (with $p>0$ ), or a cyclic group, or $G=D_{2 p^{r}}$ with $p$ odd. Then there exists a $G$-module $V$ such that $I_{H}$ is not generated by homogeneous invariants of positive degree strictly less than $|G|$.

Proof. In the proofs of the Propositions 8, 9 and 10 respectively, we constructed a $G$ module $V$ and a non-zero $v \in V$ such that $f(v)=0$ for all homogeneous $f \in \mathcal{O}(V)^{G}$ of positive degree strictly less than $|G|$, but such that there exists a homogeneous $f \in \mathcal{O}(V)^{G}$ of degree $|G|$ with $f(v) \neq 0$. This shows that $f \notin \mathcal{O}(V)_{+,<|G|}^{G} \mathcal{O}(V)$.

Now we use relative degree bounds for separating invariants and good degree bounds for generating invariants of non-modular groups, that appear as a subquotient, to get improved degree bounds for separating invariants in the modular case.

Proposition 11. Let char $K=p$ and $G$ be a finite group. Assume there exists a chain of subgroups $N \subset H \subset G$ such that $N$ is a normal subgroup of $H$ and such that $H / N$ is non-cyclic of order s coprime to $p$. Then

$$
\beta_{\mathrm{sep}}(G) \leq \begin{cases}\frac{3}{4}|G| & \text { in case } s \text { is even } \\ \frac{5}{8}|G| & \text { in case } s \text { is odd. }\end{cases}
$$

Proof. By SEzer [16], for a non-cyclic non-modular group $U$, we have $\beta(U) \leq \frac{3}{4}|U|$ in case $|U|$ is even, and $\beta(U) \leq \frac{5}{8}|U|$ in case $|U|$ is odd. We now assume $s$ is even; the other case is essentially the same. Since $\beta_{\text {sep }}(U) \leq \beta(U)$ always holds, we get by using Theorem 2

$$
\begin{gathered}
\beta_{\text {sep }}(G) \leq \beta_{\text {sep }}(H)[G: H] \leq \beta_{\text {sep }}(N) \beta_{\text {sep }}(H / N)[G: H] \\
\leq \beta(H / N)[G: H]|N| \leq \frac{3}{4}[H: N][G: H]|N|=\frac{3}{4}|G| .
\end{gathered}
$$


Example 1. Assume $p=3$ and $G=A_{4}$. The Klein four group is a non-cyclic non-modular subgroup of even order. We get $\beta_{\text {sep }}\left(A_{4}\right) \leq \frac{3}{4}\left|A_{4}\right|=9$. Application of Theorem 2 shows $\beta_{\text {sep }}\left(A_{4} \times A_{4}\right) \leq \beta_{\text {sep }}\left(A_{4}\right)^{2} \leq 81$.

Example 2. Let $D_{2 n}$ be the dihedral group of order $2 n$. We know $n \leq \beta_{\text {sep }}\left(D_{2 n}\right)$ by Corollary 3. Assume char $K=p \neq 2$ and $n=p^{r} m$ with $p, m$ coprime and $m>1$. Then $D_{2 n}$ has the non-cyclic subgroup $D_{2 m}$ of even order, so $\beta_{\text {sep }}\left(D_{2 n}\right) \leq \frac{3}{4} 2 n=\frac{3}{2} n$. So the only dihedral groups, to which the proposition above does not apply, are those of the form $D_{2 p^{r}}$, which are covered by Proposition 10 .

We end this section with two questions:

Question 1. Which finite groups $G$ satisfy $\beta_{\mathrm{sep}}(G)=|G|$ ?

Question 2. Which finite groups $G$ do not have a non-cyclic non-modular subquotient?

The dihedral groups of Proposition 10 satisfy this property, and we get $\beta_{\mathrm{sep}}(G)=$ $|G|$ for those groups. But in characteristic $2, \beta_{\text {sep }}\left(S_{3}\right)<\left|S_{3}\right|$ by Proposition 7 , so the answer to the second question only partially helps to solve the first one.

Note added in proof: The conjecture following Proposition 10 claiming that in characteristic 2 we have $\beta_{\mathrm{sep}}\left(D_{2 p}\right)=p+1$ for an odd prime $p$ was recently proved by the first author jointly with Müfit Sezer: Invariants of the dihedral group $D_{2 p}$ in characteristic two, Preprint 2010.

\section{References}

[1] W. Bosma, J. Cannon, and C. Playoust, The Magma algebra system. I. The user language, J. Symbolic Comput. 24 (1997), no. 3-4, 235-265. Computational algebra and number theory (London, 1993).

[2] R. M. Bryant and G. Kemper, Global degree bounds and the transfer principle for invariants, J. Algebra 284 (2005), no. 1, 80-90.

[3] C. de Concini and C. Procesi, A characteristic free approach to invariant theory, Advances in Math. 21 (1976), no. 3, 330-354.

[4] H. Derksen and G. Kemper, Computational invariant theory, Invariant Theory and Algebraic Transformation Groups, I, Springer-Verlag, Berlin (2002), ISBN 3-540-43476-3. Encyclopaedia of Mathematical Sciences, 130.

[5] - On global degree bounds for invariants, in Invariant theory in all characteristics, Vol. 35 of CRM Proc. Lecture Notes, 37-41, Amer. Math. Soc., Providence, RI (2004).

[6] M. Domokos and P. Hegedűs, Noether's bound for polynomial invariants of finite groups, Arch. Math. (Basel) $\mathbf{7 4}$ (2000), no. 3, 161-167.

[7] J. Draisma, G. Kemper, and D. Wehlau, Polarization of separating invariants, Canad. J. Math. 60 (2008), no. 3, 556-571.

[8] P. Fleischmann, The Noether bound in invariant theory of finite groups, Adv. Math. 156 (2000), no. $1,23-32$.

[9] J. Fogarty, On Noether's bound for polynomial invariants of a finite group, Electron. Res. Announc. Amer. Math. Soc. 7 (2001) 5-7 (electronic).

[10] G. Kemper, Computing invariants of reductive groups in positive characteristic, Transform. Groups 8 (2003), no. 2, 159-176.

[11] —, Separating invariants, J. Symbolic Comput. 44 (2009), no. 9, 1212-1222.

[12] D. Mumford, J. Fogarty, and F. Kirwan, Geometric invariant theory, Vol. 34 of Ergebnisse der Mathematik und ihrer Grenzgebiete (2) [Results in Mathematics and Related Areas (2)], Springer-Verlag, Berlin, third edition (1994), ISBN 3-540-56963-4. 
[13] P. E. Newstead, Introduction to moduli problems and orbit spaces, Vol. 51 of Tata Institute of Fundamental Research Lectures on Mathematics and Physics, Tata Institute of Fundamental Research, Bombay (1978), ISBN 0-387-08851-2.

[14] M. Roberts, On the Covariants of a Binary Quantic of the $n^{\text {th }}$ Degree, The Quarterly Journal of Pure and Applied Mathematics 4 (1861) 168-178.

[15] B. J. Schmid, Finite groups and invariant theory, in Topics in invariant theory (Paris, 1989/1990), Vol. 1478 of Lecture Notes in Math., 35-66, Springer, Berlin (1991).

[16] M. Sezer, Sharpening the generalized Noether bound in the invariant theory of finite groups, J. Algebra 254 (2002), no. 2, 252-263.

[17] T. A. Springer, Linear algebraic groups, Vol. 9 of Progress in Mathematics, Birkhäuser Boston Inc., Boston, MA, second edition (1998), ISBN 0-8176-4021-5.

[18] D. L. Wehlau, The Noether number in invariant theory, C. R. Math. Acad. Sci. Soc. R. Can. 28 (2006), no. 2, 39-62.

Zentrum Mathematik - M11, Technische Universität München, Boltzmannstrasse 3, D85748 Garching, Germany

E-mail address: kohls@ma.tum.de

Mathematisches Institut, Universität Basel, Rheinsprung 21, CH-4051 Basel, SwitzerLAND

E-mail address: Hanspeter.Kraft@unibas.ch 